\title{
Spectroscopic and photometric observations of the short-period RS CVn-type star WY Cnc ${ }^{\star}$
}

\author{
D. Kjurkchieva ${ }^{1}$, D. Marchev ${ }^{1}$, and W. Ogloza ${ }^{2}$ \\ ${ }^{1}$ Department of Physics, Shoumen University, 9700 Shoumen, Bulgaria \\ and Isaac Newton Institute of Chile, Bulgarian Branch \\ e-mail: d.kyurkchieva@shu-bg.net \\ e-mail: d.marchev@shu-bg.net \\ 2 Pedagogical University, ul. Podchorazych 2, 30-084 Cracow and Mt. Suhora Observatory, Poland \\ e-mail: ogloza@ap.krakow.pl
}

Received 7 February 2003 / Accepted 11 September 2003

\begin{abstract}
High-resolution spectroscopic observations around the $\mathrm{H}_{\alpha}$ line and BVRI photometry of the eclipsing short-period RS CVn star WY Cnc are presented. The solutions of our radial velocity curves and light curves yielded the following values for masses and radii of the components: $M_{1}=0.84 M_{\odot}, M_{2}=0.46 M_{\odot}, R_{1}=1.06 R_{\odot}, R_{2}=0.65 R_{\odot}$. The measured rotational broadenings of the spectral lines correspond to equatorial velocities $V_{1}=78 \mathrm{~km} \mathrm{~s}^{-1}$ and $V_{2}=49 \mathrm{~km} \mathrm{~s}^{-1}$. The distortions of our multicolor light curve of WY Cnc were reproduced by two cool spots on the primary star. An increase of the out-of-eclipse brightness by 0.1 mag in February 2001 was detected. Our spectra show $\mathrm{H}_{\alpha}$ line in emission from the secondary star. The chromospheric activity appeared also in the observed emission cores of the CaII H and $\mathrm{K}$ lines as well as the CaI 6494 line.
\end{abstract}

Key words. stars: activity - binaries: eclipsing - binaries: spectroscopic - stars: chromospheres - stars: individual: WY Cnc stars: starspots

\section{Introduction}

WY Cnc was discovered by Kippenhahn (1953) as an eclipsing system of Algol type. Chambliss (1965) determined a smaller ratio of the star radii than that of other Algol-type binaries and a few years later, due to the irregularities of its light curves as well as the CaII H and K emission lines (Popper 1976) WY Cnc was classified as a RS CVn, short-period system (Hall 1976). They are detached binaries with main-sequence stellar components and their photometric variability is successfully interpreted by cool spots modulating the light curve by the stellar rotation. The observations in different spectral ranges (from $\mathrm{X}$-ray to radio) indicate chromospheric-coronal radiation from these stars.

In the last three decades visible photometry of WY Cnc was made by Oliver (1974), Sarma (1976), Awadalla \& Budding (1979), Naftilan (1987), Zeilik et al. (1989), Heckert \& Zeilik (1990), Zeilik et al. (1990), Rao et al. (1991), Heckert et al. (1998). They found various peculiarities of the optical light

Send offprint requests to: W. Ogloza, e-mail: ogloza@ap. krakow.pl

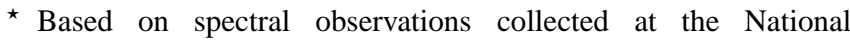
Astronomical Observatory at Rozhen, research was supported in part by the NATO Linkage grant No. PST.CLG.978810 and grant No. 1/2002 of the Shoumen University curves: asymmetric secondary eclipse; considerable increase of the brightness before the primary eclipse; small depressions before and after the secondary eclipse. The IR observations of WY Cnc showed an infrared excess of the primary star of $0.14 \mathrm{mag}$ and out-of-eclipse colors redder by $0.16 \mathrm{mag}$ than those expected from the combination of the spectral types of the components (Arevalo \& Lazaro 1990).

There is evidence for long-term variability of WY Cnc and different authors determine different periods: Oliver (1974) 5.5 yr; Sarma (1976) and Maceroni et al. (1990) - 10 yr; Rao et al. (1991) - $50 \mathrm{yr}$; Saar \& Brandenburg (1999) - $27 \mathrm{yr}$. Heckert (2001) divides the long-term light changes of WY Cnc into two types: secular luminosity increases that are superposed on longer-term slower variations.

There is agreement about the spectral type G5V of the primary star of WY Cnc while the secondary's spectral type is less well determined. Awadala \& Budding (1979) determined it as M2, Oliver (1974) supposed a subgiant K0 secondary that is the source of the distortion wave while Rao et al. (1991) derived a K9V type.

Arevalo \& Lazaro (1999) found emission excesses in the $\mathrm{H}_{\alpha}, \mathrm{H}_{\beta}$ and CaII IRT lines in all orbital phases. In the UV range WY Cnc displays strong emission in the MgII h and $\mathrm{k}$ lines (Budding et al. 1982). 
Morris \& Mutel (1988) and Drake et al. (1989) carried out radio observations of the star while Walter et al. (1980) established that it is a weak X-ray source.

The global parameters of WY Cnc are poorly determined (mainly photometrically) because the primary component contributes a high fraction of the continuum flux (about 96-99\%) and lines of the secondary star are not visible in the spectra. New radial velocity curves are needed for reliable determination of the mass ratio and the global parameters of the system. Another goal of our photometric and spectral observations of this star is an investigation of its activity.

\section{Observations}

During 5 nights at the end of 2000 (November 25, 28, 29; December 3, 6) and at the beginning of 2001 (February 11, 15 ), we carried out BVRI photometry of WY Cnc with a twochannel photometer mounted on the $60-\mathrm{cm}$ telescope (Kreiner et al. 1993) at the Mt. Suhora Observatory. The telescope is equipped with an autoguiding system (Krzesinski \& Wojcik 1993). The integration times were $20 \mathrm{~s}$ in the $B$ and $I$ filters and $15 \mathrm{~s}$ in the $V$ and $R$ filters. We used PPM 99256 (BD +271707 ) as a comparison star as well as $75 \mathrm{Cnc}$ (HD 78418) and PPM 99267 (HD 77444) as check stars. A cross-calibration of the channels for the different sensitivity was made every night.

The photometric data were phased according to the ephemeris (Kreiner et al. 2001)

$\operatorname{HJD}(\operatorname{MinI})=2446025.9001+0.82937067 * E$

and left in the instrumental system. The complete light curve is shown in Fig. 1. We found that the time of our primary minimum

$\operatorname{HJD}(\operatorname{MinI})=2451952.5707 \pm 0.0003$

precede that calculated according to the ephemeris (1) by $0.014 \mathrm{P}$.

On four nights at the beginning of April and May 2002 WY Cnc was observed also in the spectral range around the $\mathrm{H}_{\alpha}$ line (6470-6670 ̊̊) with resolution $0.19 \AA /$ pixel. We used a CCD Photometrics AT200 camera with the SITe SI003AB $1024 \times 1024$ pixels chip mounted on the Coude spectrograph (grating $B \& L 632 / 14.7^{\circ}$ ) on the 2-m telescope of the National Astronomical Observatory at Rozhen. The seeing during the observations did not exceed $2 \operatorname{arcsec}$ (FWHM). The exposure time was $20 \mathrm{~min}$. The bias frames and flat-field integrations were obtained at the beginning and end of each night. All stellar integrations were alternated with $\mathrm{Th}$-Ar comparison source exposures for wavelength calibration. The ratio $\mathrm{S} / \mathrm{N}$ was around 60 in the April run and around 90-100 in the May run. $\beta$ Vir or $16 \mathrm{Cnc} \mathrm{C}$, the radial velocity standard stars, were observed every night.

In addition, WY Cnc was observed on May 12002 during the primary eclipse in the range of the CaII $\mathrm{H}$ and $\mathrm{K}$ lines with $\mathrm{S} / \mathrm{N}$ around 15 (Fig. 2).

The spectral data were reduced in a standard way using the PCIPS (Smirnov et al. 1992) and Rewia (Borkowski 1988) software packages by bias substraction, flat-field division and

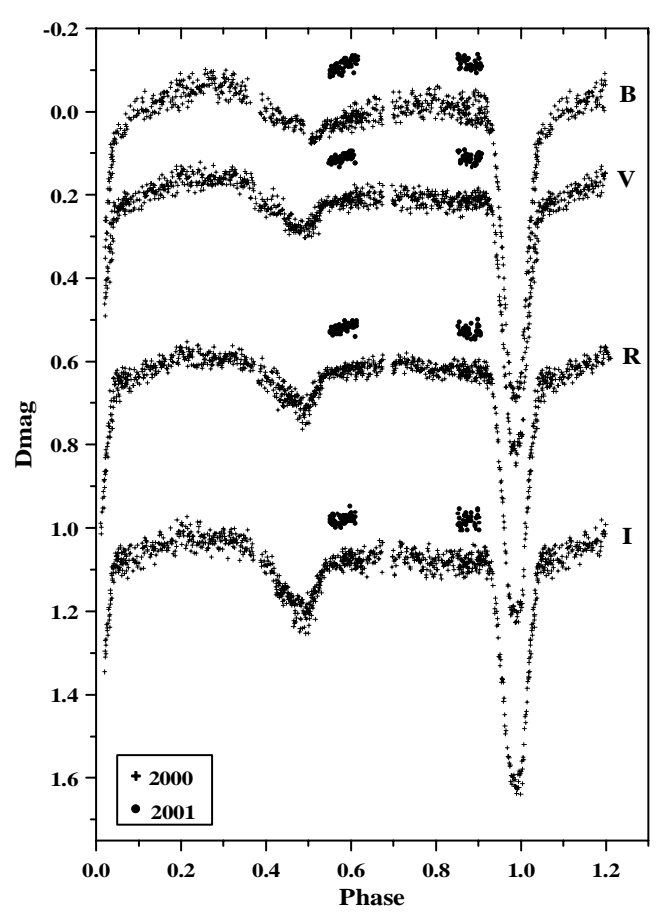

Fig. 1. Light curve of WY Cnc.

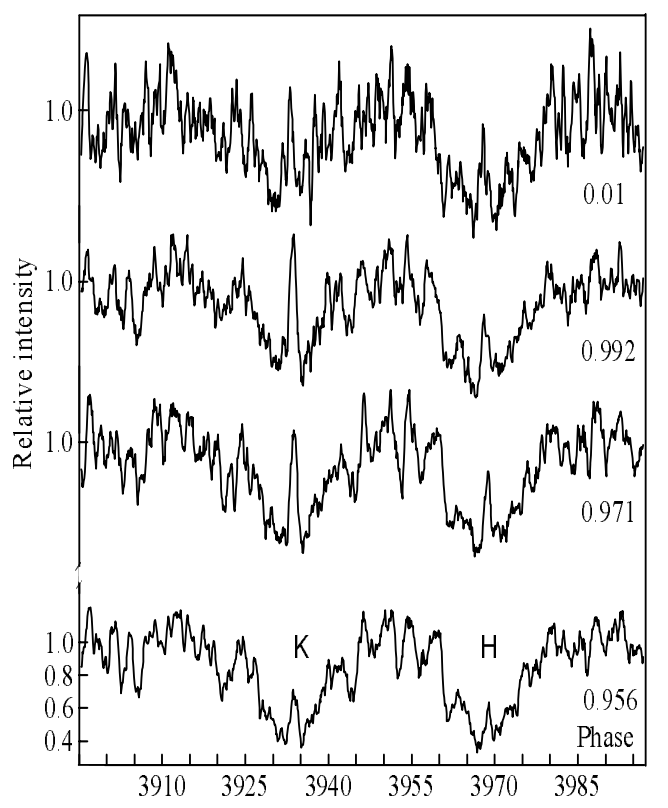

Fig. 2. CaII H and K lines of WY Cnc during the primary eclipse.

wavelength calibration. Most of the normalized spectra around the $\mathrm{H}_{\alpha}$ line are shown in Figs. 3-6. The phases in Figs. 2-6 are corrected by +0.014 in respect to the ephemeris (1).

\section{Analysis of the spectral data}

\subsection{Orbital variability of the spectral lines}

The analysis of the orbital variability of the spectra yields information about the dominant sources of the spectral lines as well as the locations of the active regions in binary systems. 


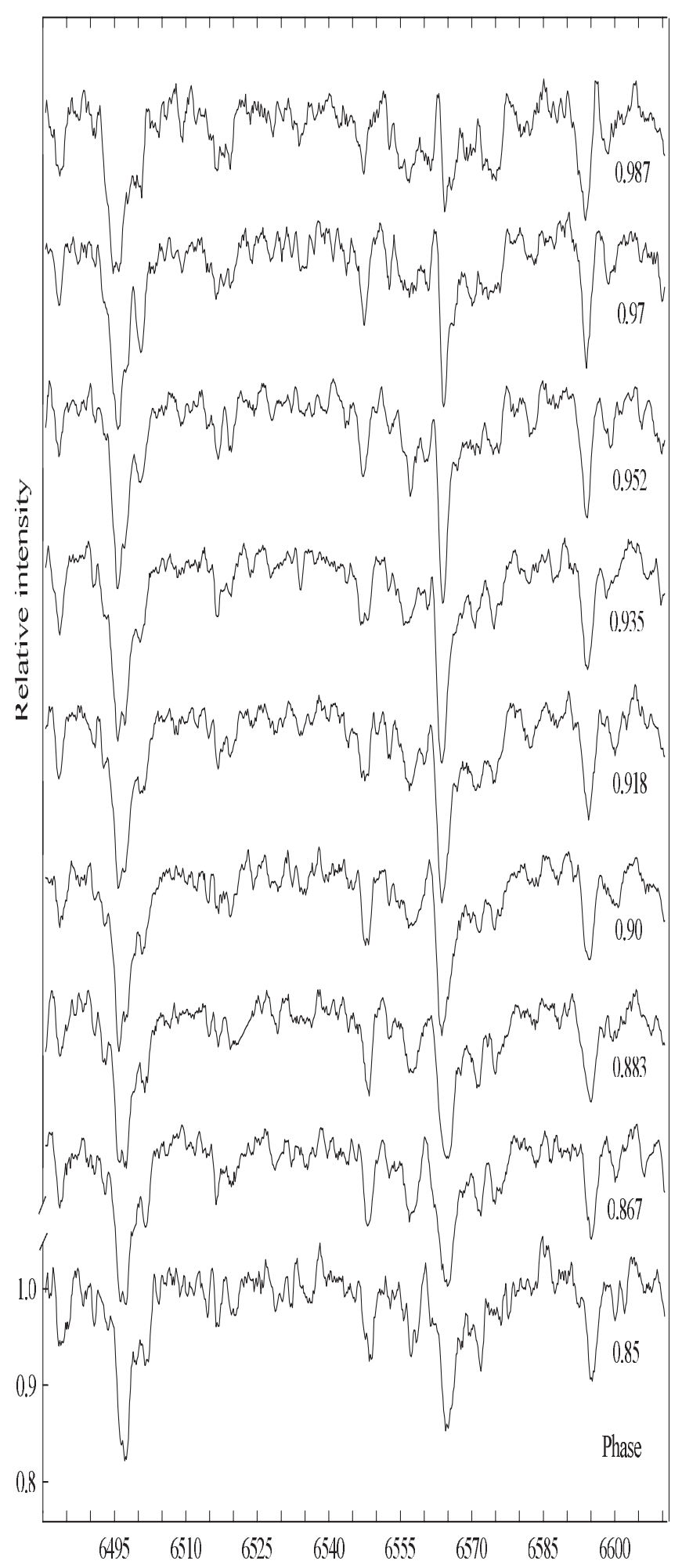

Fig. 3. The spectra of WY Cnc from April 12002.

We observed WY Cnc spectroscopically around the $\mathrm{H}_{\alpha}$ line because it is a spectroscopic indicator of chromospheric activity (Zarro \& Rogers 1983; Herbig 1985; Frasca \& Catalano 1994; Strassmeier et al. 1990). The $\mathrm{H}_{\alpha}$ emission excess in active stars may appear as an $\mathrm{H}_{\alpha}$ line above the continuum or as a weak absorption line with filled-in core.

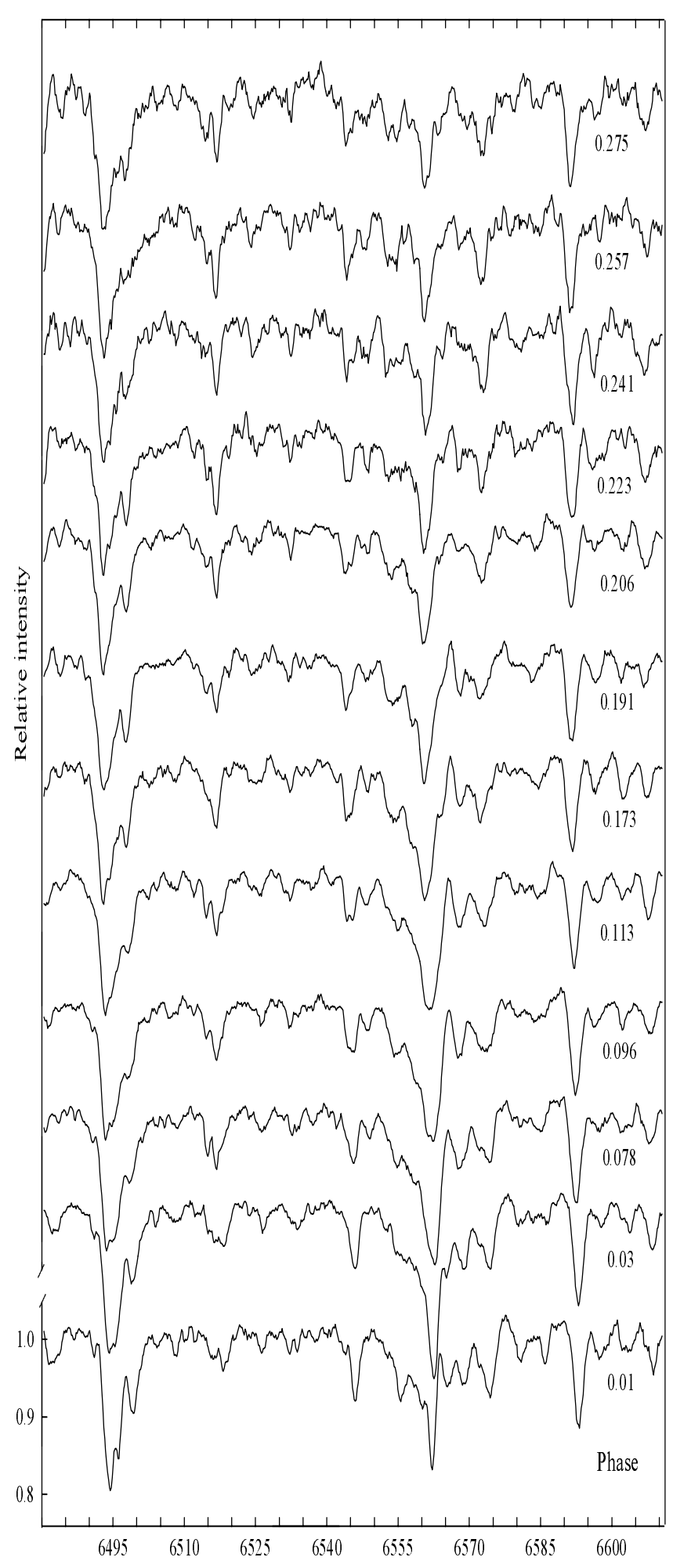

Fig. 4. The spectra of WY Cnc from April 22002.

During most orbital phases the observed $\mathrm{H}_{\alpha}$ absorption line of WY Cnc is asymmetric with variable profile. Before the primary eclipse an emission feature is visible on the blue wing of the main absorption profile (Fig. 3). It shifts in the direction expected from the lines of the secondary star. The emission feature appears on the red wing of the main absorption profile after the primary eclipse (Fig. 4) and shifts to longer 


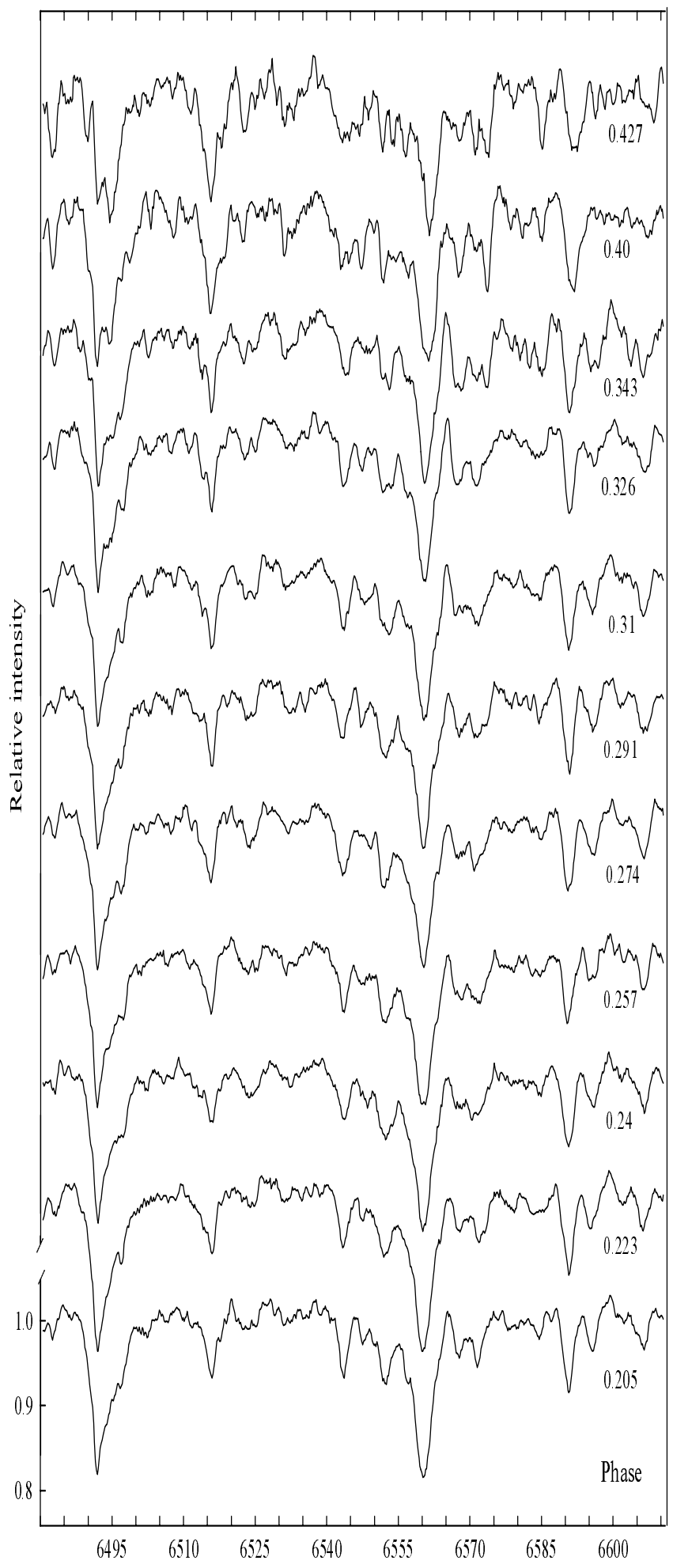

Fig. 5. The spectra of WY Cnc from May 2002.

wavelengths until the first quadrature, i.e. also in the expected direction of the lines of the secondary star. After that the shift of the emission feature is opposite until the secondary eclipse (Fig. 5). In the phase range $0.47-0.54$ the absorption $\mathrm{H}_{\alpha}$ profile is symmetric (Fig. 6). After the secondary eclipse the emission feature appears in the blue wing of the absorption line.

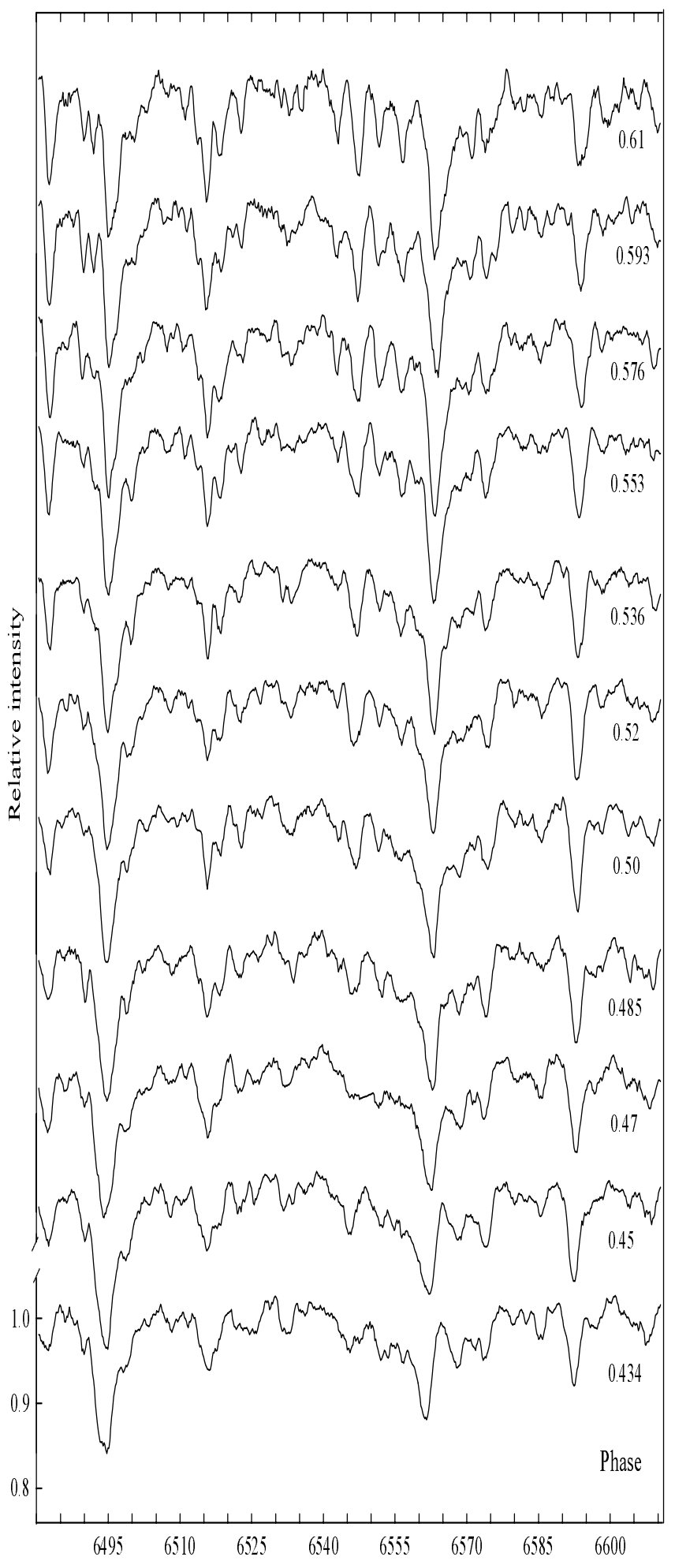

Fig. 6. The spectra of WY Cnc from May 32002.

Hence our spectral data show directly the $\mathrm{H}_{\alpha}$ line in emission from the secondary component of WY Cnc. Arevalo \& Lazaro (1999) inferred the same conclusion by the method of spectral subtraction. They obtain an emission feature which changes position relative to the $\mathrm{H}_{\alpha}$ absorption line and attributed it to the cool secondary star of WY Cnc. 


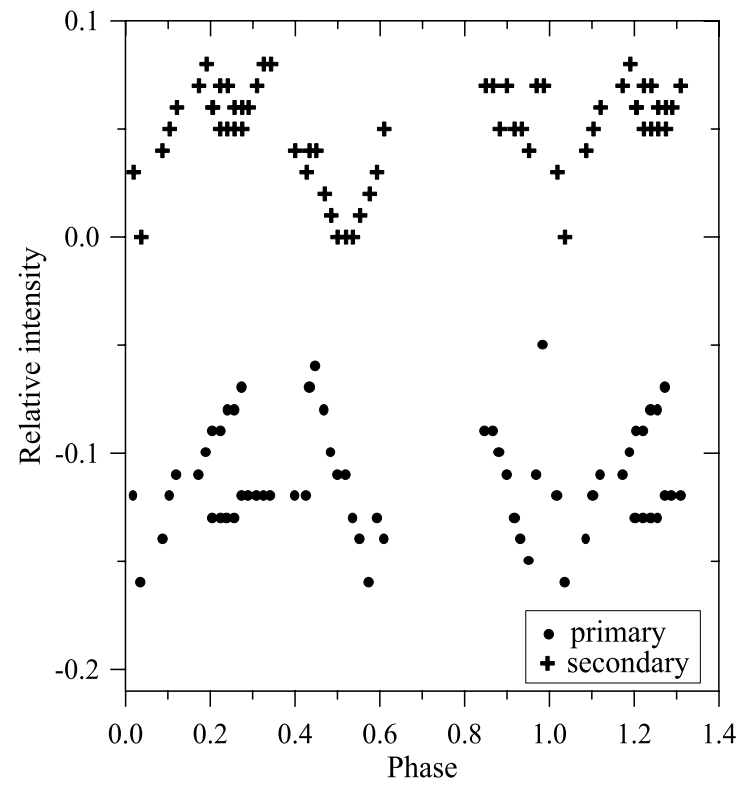

Fig. 7. The relative changes of the strength of the $\mathrm{H}_{\alpha}$ spectral features of the stellar components of WY Cnc.

That the secondary star shows an $\mathrm{H}_{\alpha}$ emission line is not unexpected, taking into account its late spectral type. Montes et al. (1995) found that some stars cooler than $5000 \mathrm{~K}$ show $\mathrm{H}_{\alpha}$ lines above the continuum.

In our spectra the $\mathrm{H}_{\alpha}$ line of the secondary star is in emission above the continuum level at most phases outside the eclipses. At the rest phases it appears as a peak moving on the wide wings of the absorption $\mathrm{H}_{\alpha}$ profile of the primary star. That is why we assumed that the level of the wide wings of the absorption $\mathrm{H}_{\alpha}$ profile ( 0.05 below the continuum) is an appropriate initial zero-level for the measurement of the relative changes of the strength of the $\mathrm{H}_{\alpha}$ spectral features of the stellar components of WY Cnc. The crosses in Fig. 7 present the heights of the $\mathrm{H}_{\alpha}$ emission line of the secondary star and the dots show the depths of the $\mathrm{H}_{\alpha}$ absorption line of the primary star with respect to the zero-level we chose.

One can see the following trends of the $\mathrm{H}_{\alpha}$ line of the secondary star (Fig. 7): (a) The emission feature disappears in the middle of the secondary eclipse when its source (the secondary star) is invisible; (b) The emission gradually increases before and after the secondary eclipse and stays almost constant outside the eclipses; (c) The emission during the primary eclipse is smaller than at the out-of-eclipse phases.

We note the following trends in the phase behavior of the absorption $\mathrm{H}_{\alpha}$ line: (a) Mirror symmetry of its shape at the phases symmetric to the middle of the primary eclipse - its red wing is steeper at phases $0.08-0.1$ whereas the blue wing is steeper at the phases 0.9-0.935 (Figs. 3, 4); (b) The profile is slightly wider, with almost filled-in core at the symmetric (in respect to the eclipse) phases $0.867-0.883$ and $0.096-0.113$; (c) The absorption profile becomes narrower in the middle of the primary eclipse; (d) The depths of the absorption feature considerably decreased on April 2 around the first quadrature. This may be considered another confirmation of the common trend of the spectral lines of the short-period RS CVn-stars to be more stronger around the second than around the first quadrature (so called "second quadrature effect", Kjurkchieva et al. 2002).

The phase variability of the strength (depth) of the $\mathrm{H}_{\alpha}$ line of the primary star is not as regular as that of the secondary (Fig. 7). Its changes should be symmetric with respect to the middle of the secondary eclipse but a decrease of the line depth from the beginning to the end of this eclipse is visible. It is difficult to find also some trend of the changing of the depth of the line from the primary during the primary eclipse.

A second emission feature appears between the absorption $\mathrm{H}_{\alpha}$ line of the primary star and the emission line of the secondary star in the phase range $0.206-0.275$ on April 2 but it is not visible on May 2 at the same phases. This additional emission feature may be the reason for the unusually shallow, distorted $\mathrm{H}_{\alpha}$ absorption line of the primary star on April 2. Arevalo \& Lazaro (1999) also detected an additional $\mathrm{H}_{\alpha}$ emission component in the spectra of WY Cnc and attributed it to extended structure between the stars.

CaI 6494 is the strongest line of WY Cnc in the observed spectral range $6470-6670 \AA$. We found orbital variability of this line also in the short-period RS CVn-stars CG Cyg (Kjurkchieva et al. 2003a) and ER Vul (Kjurkchieva et al. 2003b). In their spectra the CaI 6494 profiles were slightly weaker than $\mathrm{H}_{\alpha}$ and their cores were filled-in by emission. The $\mathrm{CaI} 6494$ line is stronger in the spectra of WY Cnc but the emission excess in its core is not so apparent. The profiles of the line from the primary star are strongly asymmetric between the primary and secondary eclipse and those of the secondary star are hardly seen (they are visible only after the secondary eclipse and before the primary one).

The observed CaII H (3968.47 ̊) and K (3933.66 ̊) lines (Fig. 2) have widths around $20 \AA$ and $13 \AA$ respectively and almost equal depths ( 3 times deeper than the $\mathrm{H}_{\alpha}$ and FeI lines). The lines $\mathrm{H}$ and $\mathrm{K}$ have sharp emission cores typical for the active RS CVn stars. They are strongest in the middle of the eclipse when the emission core of the $\mathrm{K}$ line is above the continuum level. The emission core of the $\mathrm{K}$ line is narrower, more symmetric and about twice as strong as that of the $\mathrm{H}$ line. There is a small additional emission feature just at the red end of the $\mathrm{H}$ core. Self-reversal features at the top of the $\mathrm{H}$ and $\mathrm{K}$ emission cores are not visible during the primary eclipse we observed. The $\mathrm{K}$ emission core strengthens almost 3 times in the phase range $0.95-0.99$ and splits at the phase 0.01 . The $\mathrm{H}$ emission core also increases around 2 times in the middle of the eclipse.

\subsection{Radial velocity solution}

The measurement of the radial velocity of the spectral lines of the short-period RS CVn-stars is difficult due to their rotational broadening and variable blending with the surrounding metal lines (Frasca et al. 2000). Moreover the profiles are distorted by emission or absorption features. Nevertheless Hill et al. (1989) argued that for detached systems with rotational velocity $<100 \mathrm{~km} \mathrm{~s}^{-1}$ (like WY Cnc) the Gaussian approximation is very good. 


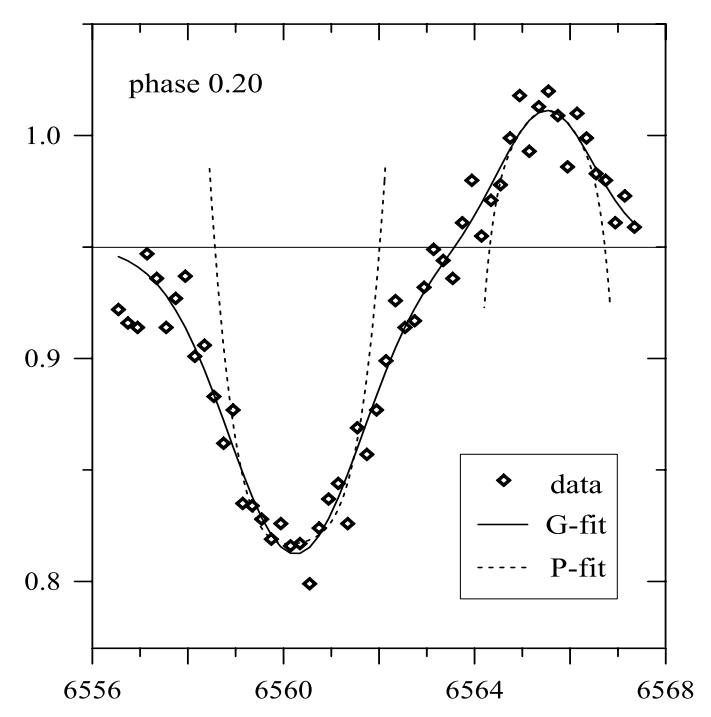

Fig. 8. The observed $\mathrm{H}_{\alpha}$ profile both with Gaussian fit (G-fit) for the radial velocity measurement and polynomial fit (P-fit) for the equatorial velocity measurement.

Another considerable complication in the case of WY Cnc is the negligible contribution of its secondary component to the total luminosity of the system that correspondingly leads to invisibility of its lines in the spectra. So, WY Cnc has traditionally been classified as single-lined. Only recently Pojmanski (1998) and Arevalo \& Lazaro (1999) detected indirectly spectral lines of the secondary star (by the method of spectral substraction).

The $\mathrm{H}_{\alpha}$ line of the secondary star of WY Cnc is directly visible as an emission peak in our spectra. Thus, because of the contrast this spectral feature is well detectable (Figs. 3-6) and might be used also for radial velocity measurements.

We measured the radial velocities of the lines $\mathrm{H}_{\alpha}$, FeI 6593 and $6547 \AA$ by fitting their profiles with the sums of two Gaussians at each phase. Figure 8 illustrates this procedure for the $\mathrm{H}_{\alpha}$ line. The errors of the radial velocity of the common fit do not exceed $19 \mathrm{~km} \mathrm{~s}^{-1}$ for the lines of the primary star and $34 \mathrm{~km} \mathrm{~s}^{-1}$ for the lines of the secondary star. The obtained values are given in Table 1 . The radial velocity data were fitted by sinusoids (Fig. 9) and the solution of the radial velocity curves is: $K_{1}=88.3 \pm 1.2 \mathrm{~km} \mathrm{~s}^{-1} ; K_{2}=160 \pm 1.9 \mathrm{~km} \mathrm{~s}^{-1}$.

Our $K_{1}$ value is close to those obtained by Awadalla \& Budding (1979) $K 1=92.8 \pm 28 \mathrm{~km} \mathrm{~s}^{-1}$ and Pojmanski (1998) $K 1=93 \mathrm{~km} \mathrm{~s}^{-1}$. Our $K_{2}$ value is smaller than the only known value of $181 \mathrm{~km} \mathrm{~s}^{-1}$ (Pojmanski 1998).

We determined the rotation broadening of the investigated lines with the same procedure as that used for the star SV Cam (Kjurkchieva et al. 2002), i.e. by fitting the central parts of their profiles with 6th-order polynomials and measuring the half width of these fits at the continuum level (Fig. 8). The obtained values correspond to equatorial velocities $V_{1}=78 \pm 5 \mathrm{~km} \mathrm{~s}^{-1}$ and $V_{2}=49 \pm 5 \mathrm{~km} \mathrm{~s}^{-1}$. Pojmanski (1998) found $V_{1} \sin i=$ $75 \mathrm{~km} \mathrm{~s}^{-1}$ while Arevalo \& Lazaro (1999) determined considerably smaller values: $V_{1}=58 \mathrm{~km} \mathrm{~s}^{-1}$ and $V_{2}=34 \mathrm{~km} \mathrm{~s}^{-1}$.
Table 1. Radial velocity data in $\mathrm{km} \mathrm{s}^{-1}$.

\begin{tabular}{|c|c|c|c|c|}
\hline phase & $\overline{V_{1}\left(\mathrm{H}_{\alpha}\right)}$ & $\overline{V_{2}\left(\mathrm{H}_{\alpha}\right)}$ & $\bar{~} V_{1}(\mathrm{Fe})$ & $\overline{V_{2}(\mathrm{Fe})}$ \\
\hline 0.850 & 89.1 & -139.4 & & \\
\hline 0.867 & 80.0 & -130.3 & & \\
\hline 0.883 & 70.8 & & & \\
\hline 0.900 & & -84.6 & 65.1 & \\
\hline 0.918 & & -75.4 & 60.5 & \\
\hline 0.935 & & -66.3 & 51.4 & \\
\hline 0.952 & & -48.0 & 42.3 & \\
\hline 0.970 & & -29.7 & & \\
\hline 0.987 & & -2.3 & & \\
\hline 0.011 & & & 3.6 & \\
\hline 0.029 & -13.7 & & & \\
\hline 0.113 & -50.3 & 123.4 & & \\
\hline 0.173 & & 141.7 & -55.5 & 162.9 \\
\hline 0.19 & & 150.8 & -64.6 & 162.9 \\
\hline 0.206 & & 150.8 & -69.1 & \\
\hline 0.224 & & 160.0 & -73.7 & \\
\hline 0.241 & & & & 153.8 \\
\hline 0.257 & & 169.1 & -73.7 & \\
\hline 0.275 & & 160.0 & -73.7 & \\
\hline 0.205 & -82.0 & 146.6 & -78.3 & 149.2 \\
\hline 0.223 & -86.6 & 151.1 & -78.3 & \\
\hline 0.240 & -91.1 & 151.1 & -82.8 & 158.3 \\
\hline 0.256 & -86.6 & 155.7 & -82.8 & 162.9 \\
\hline 0.274 & -82.0 & 155.7 & -82.8 & 158.3 \\
\hline 0.290 & -82.0 & 151.1 & -82.8 & 149.2 \\
\hline 0.310 & -77.4 & 142.0 & -78.3 & 144.7 \\
\hline 0.326 & -72.8 & 142.0 & -73.7 & \\
\hline 0.343 & -68.3 & 132.8 & -73.7 & 135.7 \\
\hline 0.400 & -50.0 & & -23.6 & \\
\hline 0.427 & -27.1 & 105.4 & -23.6 & \\
\hline 0.434 & -54.8 & & -23.6 & \\
\hline 0.450 & -22.8 & & -19.1 & \\
\hline 0.470 & -13.7 & & -5.4 & \\
\hline 0.485 & 0.3 & & 3.6 & \\
\hline 0.500 & 13.7 & & 8.2 & \\
\hline 0.520 & 9.1 & & 12.7 & \\
\hline 0.536 & 18.3 & & 21.8 & \\
\hline 0.553 & 22.8 & & 26.4 & \\
\hline 0.576 & 27.4 & & 40.0 & \\
\hline 0.593 & 45.7 & & 44.6 & -77.0 \\
\hline 0.610 & & & 67.3 & \\
\hline
\end{tabular}

\section{Light curve solution}

Our multicolor light curve of WY Cnc shows the following peculiarities (Fig. 1):

(a) the brightness between the secondary and primary eclipse remains almost constant and smaller than that around the first quadrature;

(b) the secondary eclipse is asymmetric;

(c) there is some weak increase of the brightness before the primary eclipse;

(d) there are small depressions before and after secondary eclipse.

The last three peculiarities are similar to those observed in the IR range by Arevalo \& Lazaro 1990).

For the light curve modeling we used the standard package of the Wilson-Devinney code (Wilson \& Devinney 1971; 


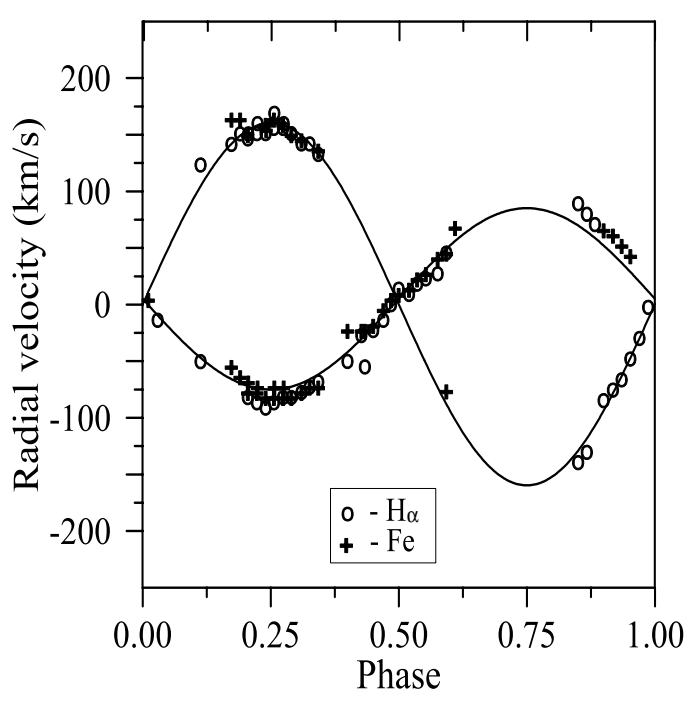

Fig. 9. Radial velocity curves of WY Cnc.

Wilson 1993) and the light-fitting procedure of Budding \& Zeilik (1987).

The aim of the first step of the procedure was to reproduce those parts of the light curve that seem "regular" (unaffected by the presence of spots) varying the star parameters. The regular parts of our light curve are the primary symmetric eclipse and the steeper branch of the secondary eclipse. The only fixed parameter was the mass ratio $q$ (determined by our spectral data). We used as start values of the fitting procedure the average values of the star parameters from the previous light curve solutions (Table 2). Varying these parameters by $\pm 15 \%$ around the start values we obtained a good approximation of our photometric data in all colors for the following values: orbital inclination $i=88.0 \pm 0.1^{\circ}$; relative star radii $r_{1}=0.262 \pm 0.002$ and $r_{2}=0.162 \pm 0.002 ;$ star temperatures $T_{1}=5500 \pm 50 \mathrm{~K}$ and $T_{2}=4000 \pm 50 \mathrm{~K}$.

The second step of our fitting procedure was to search for a fit of the whole observed light curve. Usually the distortions of the light curves of the RS CVn-type stars are reproduced by cool spots on the primary star that is much more luminous than the secondary. The star WY Cnc is such a case, and thus, taking into account the distortions of our light curve, we assumed two cool spots on the primary star. Varying their parameters we obtained a good fit to the distortion curve for the following values of the spot parameters (according to the W-D definition): longitudes (the angle measured from the meridian along the line connecting the mass centers as seen from the upper poles) $220^{\circ}$ and $345^{\circ}$; colatitudes (the angle measured from the upper pole) $60^{\circ}$ and $144^{\circ}$; angular radii $19^{\circ}$ and $24^{\circ}$; equal temperatures of $4950 \mathrm{~K}$. It should be noted that for $90^{\circ}$ (or close) inclination there is a latitude ambiguity in that it is impossible to differentiate between the northern and southern star hemispheres. The obtained two spots cover around $6.4 \%$ of the primary star surface. The final result of our fitting procedure is shown in Fig. 10.

This can be compared to the following results. Zeilik et al. (1990) fitted the 1978-1989 distortion light curves of WY Cnc by black $(0 \mathrm{~K})$ spots at low latitudes on the primary star, with angular size $6-13^{\circ}$ that tend to fall into two active longitude belts (ALBs) near the quadratures. Rao et al. (1991) reproduced the 1973-1986 distortion light curve by four dark spots with angular sizes $3-24^{\circ}, 700-800 \mathrm{~K}$ cooler than surrounding photosphere and located at middle latitudes. Heckert et al. (1998) reproduced the 1988-1997 light curves by two cool spots at different locations on the primary star with sizes $7-35^{\circ}$ and found that the $270^{\circ}$ ALB tends to confine the spots fairly well but the $90^{\circ}$ ALB does not. Heckert (2001) modelled the last 19972000 light curves by 2 cool spots with sizes $4-26^{\circ}$.

\section{Global parameters of WY Cnc}

Using our values of $K_{1}$ and $K_{2}$ and the value of the orbital inclination $i=88^{\circ}$ determined photometrically we obtained the masses of the star components of WY Cnc to be $M_{1}=$ $0.84 \pm 0.04 M_{\odot}$ and $M_{2}=0.46 \pm 0.03 M_{\odot}$.

On the basis of our radial velocity solution and relative star radii $r_{1}=0.26$ and $r_{1}=0.16$ from the light curve solution we calculated the absolute star radii: $R_{1}=1.06 \pm 0.05 R_{\odot}$ and $R_{2}=0.65 \pm 0.04 R_{\odot}$ with $R_{2} / R_{1}=0.613$. We should point out that the ratio of the equatorial velocities $V_{2} / V_{1}=0.63$ we obtained on the basis of the measured rotational broadenings of the spectral lines is close to the stellar radii ratio $k=0.613$.

On the mass-radius relation for MS stars, the radii of the star components of WY Cnc corresponding to the values of the stellar masses obtained by us are $R_{1}=0.86 R_{\odot}$ and $R_{2}=$ $0.52 R_{\odot}$. Thus our observations lead to the conclusion that the two star components of WY Cnc are oversized for their masses. The ratio of radii of the primary and secondary star to the radii of their Roche lobes are 0.65 and 0.52 respectively, i.e. the two components fill-in considerable part of their Roche lobes.

Table 2 summarizes the global parameters of the binary system WY Cnc determined by different authors.

On the basis of the determined radii and temperatures of the stellar components we obtained the luminosity and the absolute visual magnitude of the system WY Cnc $L=0.90 L_{\odot}$ and $M=$ 4.82. These values yield a distance of $73 \mathrm{pc}$, equal to the value of the Hipparcos catalogue (Perryman et al. 1997) but smaller than the value $160 \mathrm{pc}$ of the Catalogue of the chromospheric active binary systems (Strassmeier et al. 1988).

The observed CaII $\mathrm{K}$ and $\mathrm{H}$ lines can be used also for the determination of the absolute visual magnitude of WY Cnc by the Wilson-Bappu relations (Montes et al. 1994):

$M_{V}=-14.8 \log W_{0}(\mathrm{~K})+29.87$

and

$M_{V}=-16.8 \log W_{1}(\mathrm{~K})+41.88$.

Here $W_{0}(\mathrm{~K})$ and $W_{1}(\mathrm{~K})$ are the full width at half intensity of the emission core (so called Wilson-Bappu width) and the full width of the emission core respectively; these values are corrected for the instrumental width (Montes et al. 1994) and expressed in $\mathrm{km} \mathrm{s}^{-1}$.

Table 3 lists the measured $W_{0}(\mathrm{~K})$ and $W_{1}(\mathrm{~K})$ as well as the equivalent width $E W$ (all in $\AA$ ) from our spectra.

Substituting our values $W_{0}(\mathrm{~K})=60 \mathrm{~km} \mathrm{~s}^{-1}$ and $W_{1}(\mathrm{~K})=$ $195 \mathrm{~km} \mathrm{~s}^{-1}$ in Eqs. (3) and (4) we calculated values of 3.55 and 
Table 2. Global parameters of WY Cnc.

\begin{tabular}{llllrllrrrr}
\hline \hline$i$ & $q$ & $r_{1}$ & $r_{2}$ & $T_{1}$ & $T_{2}$ & $L_{1}$ & $R_{1}$ & $M_{1}$ & $M_{2}$ & Ref. \\
\hline 86 & 0.59 & 0.25 & 0.16 & 5500 & 4000 & $0.96(V)$ & 1.03 & 0.85 & 0.50 & 1 \\
87 & & 0.24 & 0.14 & 5500 & 4000 & & & & & 2 \\
90 & 0.38 & 0.24 & 0.15 & 5520 & 4000 & $0.98(V)$ & 0.94 & 0.82 & 0.31 & 3 \\
89 & & 0.24 & 0.14 & 5500 & 3500 & $0.85(J)$ & & & & 4 \\
90 & 0.38 & 0.24 & 0.15 & 5500 & 4000 & $0.97-0.98(V)$ & 0.93 & 0.81 & 0.31 & 5 \\
90 & 0.51 & & & & & & 1.24 & 1.17 & 0.60 & 6 \\
& 0.59 & & & & & & & 0.90 & 0.53 & 7 \\
90 & 0.57 & 0.25 & 0.15 & 5520 & 3480 & 0.99 & & 0.93 & 0.53 & 8 \\
89.6 & 0.586 & 0.241 & 0.157 & 5520 & 3740 & 0.96 & 0.99 & 0.86 & 0.51 & 9 \\
90 & $0.1-0.66$ & $0.24-0.25$ & 0.15 & & & & $0.94-0.98$ & & & 10 \\
88 & 0.552 & 0.26 & 0.16 & 5500 & 4000 & 0.94 & 1.06 & 0.84 & 0.46 & 11 \\
\hline
\end{tabular}

Reference: 1 - Budding \& Zelik (1987); 2 - Arevalo (1994); 3 - Zeilik et al. (1990) ; 4 - Arevalo \& Lazaro (1990); 5 - Heckert et al. (1998); 6 - Pojmanski (1998); 7 - Arevalo \& Lazaro (1999); 8 - Awadala \& Budding (1979); 9 - Rao et al. (1991); 10 - Heckert (2001); 11 - this paper.

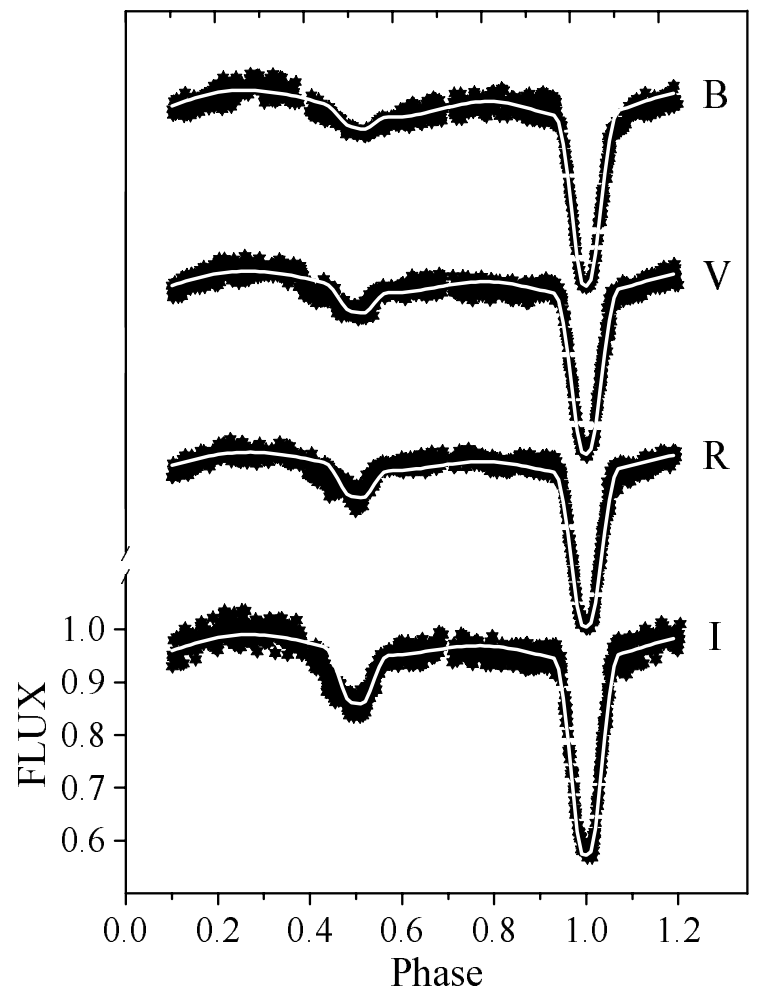

Fig. 10. Light curve approximation.

Table 3. Parameters of CaII H and K lines of WY Cnc.

\begin{tabular}{lllllll}
\hline \hline phase & $E W(\mathrm{~K})$ & $W_{1}(\mathrm{~K})$ & $W_{0}(\mathrm{~K})$ & $E W(\mathrm{H})$ & $W_{1}(\mathrm{H})$ & $W_{0}(\mathrm{H})$ \\
\hline 0.956 & 0.47 & 2.8 & 1.2 & 0.37 & 2.5 & 1.1 \\
0.971 & 0.78 & 2.8 & 1.2 & 0.61 & 2.6 & 0.8 \\
0.992 & 0.97 & 2.8 & 1.2 & 0.60 & 3.0 & 1.5 \\
0.010 & 1.15 & 2.9 & 1.3 & 0.96 & 3.5 & 1.8 \\
\hline
\end{tabular}

3.99 respectively for the absolute visual magnitude $M_{V}$. The difference between these values and the value 4.82 determined by the global star parameters (radius and temperature) may be considered acceptable, taking into account the low correlation coefficient of the Wilson-Bappu relations.
Table 4. Depth of the $\mathrm{H}_{\alpha}$ line of the primary components of RS CVn's out the eclipses.

\begin{tabular}{lllllll}
\hline \hline star & XY UMa & SV Cam & RT And & ER Vul & CG Cyg & WY Cnc \\
\hline$T$ & 5780 & 5800 & 6150 & 6000 & 5200 & 5500 \\
$d_{\text {obs }}$ & 0.31 & 0.32 & 0.28 & 0.16 & 0.12 & 0.12 \\
$d_{\text {inh }}$ & 0.326 & 0.35 & 0.35 & 0.24 & 0.15 & 0.126 \\
\hline
\end{tabular}

\section{Activity of the star}

Different indicators of stellar activity are introduced by analogy to the Sun. The large variety of the stars makes it possible to search for a relationship between the spatial, time and energetic scales of their level of activity and the global parameters (for instance the Wilson-Bappu effect). On the other hand the study of stellar activity is a basis for the improvement of the magnetic-dynamo theory and for the establishment of criteria for a solar forecast.

Emission cores in the CaII $\mathrm{H}$ and $\mathrm{K}$ lines are the primary optical indicators of chromospheric activity. They are present in our spectra of WY Cnc and are quite strong: in the middle of the primary eclipse the $\mathrm{K}$ emission core is considerably above the continuum.

The high level of star activity can be also inferred from the presence of $\mathrm{H}_{\alpha}$ emission excess. It may appear as pure emission or as filled-in $\mathrm{H}_{\alpha}$ cores. Our spectral data provide clear evidence that the $\mathrm{H}_{\alpha}$ line of the secondary star of WY Cnc is in emission, and this is a sign of its chromospheric activity. On the other hand the $\mathrm{H}_{\alpha}$ absorption line from the primary star of WY Cnc is weak for its spectral type. This can easily be seen from Table 4, which presents the temperatures of the primary stars and depths of their $\mathrm{H}_{\alpha}$ lines (measured values $d_{\text {obs }}$ as well as the corrected $d_{\text {inh }}$ for the presence of the secondary star) of the short-period RS CVn stars we observed with the same equipment.

We suppose that the weak absorbtion $\mathrm{H}_{\alpha}$ line of the primary star of WY Cnc is due to the presence of a chromospheric emission excess.

The filled-in cores of the line CaI 6494, the strongest line in the spectral range observed by us, could be considered another sign of the chromospheric activity. 
We consider the presence of an additional (second) $\mathrm{H}_{\alpha}$ emission feature at some phases outside the eclipses as well as the redder colors of the system WY Cnc as indications for the presence of matter outside stellar components of the binary. It probably is a result of mass losses during some active processes.

Our photometric observations of WY Cnc during February 2001 revealed a brightening of the star by around $0.1 \mathrm{mag}$ without its colors changing. Similar irregular increases of the brightness of WY Cnc have been noted in 1988 and 1997. Heckert (2001) called them "secular luminosity increases" and interpreted these events as the onset of a new spot cycle, assuming that the direction of longitudinal spot migration reverses for each cycle.

Although the mechanism of this luminosity increase is still not clear one should assume that whatever would be the reason for the considerable, relative stable increase of the brightness of WY Cnc should be related with the primary star. In the opposite case, due to the small contribution of the secondary star huge changes of its parameters would be necessary to reproduce the observed effect.

Different mechanisms (pulsations, mass ejection, new spot cycle) have been considered for an explanation of this phenomenon but they all have problems. We assume that the equal color indices during the two states "bright" and "normal" (noted also by Heckert et al. 1998) mean that the reason for the brightening probably is not an increase of the temperature of the primary star but rather an enlarging of its surface. Sporadic ejection of material from the primary star is one possible explanation of the observed occasional brightening of WY Cnc. Its further cooling and condensing into dust grains may be a reason for the redder colors of WY Cnc during the "normal state". But it is not clear why this material stays so long around the star. Future simultaneous photometric, spectroscopic and polarimetric observations during the secular luminosity increases could throw more light on this phenomenon.

\section{Conclusion}

The main results of our spectroscopic and photometric observations of WY Cnc and their analysis might be summarized as follows:

(1) By simultaneous solution of the radial velocity curves and the multicolor light curve we determined the global parameters of the binary system (masses, radii, temperatures, equatorial velocities, orbital inclination). The obtained values of the masses $\left(M_{1}=0.84 M_{\odot}\right.$ and $\left.M_{2}=0.46 M_{\odot}\right)$ and the radii $\left(R_{1}=1.06 R_{\odot}\right.$ and $\left.R_{2}=0.65 R_{\odot}\right)$ of the components of WY Cnc mean that they are oversized and fill in considerable parts of their Roche lobes;

(2) Besides confirmation of the known traditional appearances of activity, CaII $\mathrm{H}$ and $\mathrm{K}$ emission and $\mathrm{H}_{\alpha}$ emission excess, our observations provided additional signs for the activity of WY Cnc: $\mathrm{H}_{\alpha}$ emission line of the secondary star, filled-in cores of CaI 6494 line as well as a new secular luminosity increase in February 2001. Systematic observations in the future are necessary to explain this irregular brightening.

\section{References}

Arevalo, M. 1994, Ph.D. Thesis, Univ. La Laguna

Arevalo, M., \& Lazaro, C. 1990, AJ, 99, 983

Arevalo, M., \& Lazaro, C. 1999, AJ, 118, 1015

Awadalla, N., \& Budding, E. 1979, Ap\&SS, 63, 479

Borkowski, G. 1988, Internal report of Astron. Inst. in Torun, Poland

Budding, E. Kadouri, T., \& Gimenez, A. 1982, Ap\&SS, 88, 453

Budding, E., \& Zeelik, M. 1987, ApJ, 319, 827

Chambliss, C. 1965, AJ, 70, 741

Drake, S., Simon, T., \& Linsky, J. 1989, ApJS, 71, 905

Frasca, A., Marino, C., Catalano, S., \& Marilli, E. 2000, A\&A, 358, 1007

Frasca, A., \& Catalano, S. 1994, A\&A, 284, 883

Hall, D. 1976, in Multiple Periodic Variable Stars, ed. W. Fitch (Dordrecht: Reidel), IAU Colloq., 29, 278

Heckert, P., \& Zeilik, M. 1990, IBVS, No. 3416

Heckert, P. A., Maloney, G. V., Stewart, M. C., et al. 1998, AJ, 115, 1145

Heckert, P. 2001, AJ, 121, 1076

Herbig, G. 1985, ApJ, 289, 269

Hill, G., Fisher, W., \& Holmgren, D. 1989, A\&A, 218, 152

Kjurkchieva, D., Marchev, D., \& Zola, S. 2002, A\&A, 386, 548

Kjurkchieva, D., Marchev, D., \& Ogloza, W. 2003a, A\&A, 400, 623

Kjurkchieva, D., Marchev, D., \& Zola, S. 2003b, A\&A, 404, 611

Kippenhahn, R. 1953, Nachr. Astr. Zentralstelle 7th Jahr, No. 3, 12

Kreiner, J., Krzesinski, J., Pokrzywka, B., et al. 1993, Poster Papers on Stellar photometry, ed. I. Elliot \& C. Buttler (Dublin Institute for Advanced Studies), IAU Coll. 136, 80

Kreiner, J., Kim, C., \& Nha Il-Seong 2001, An Atlas of O-C Diagrams of Eclipsing Binary Stars (Krakow Pedagogical University Press)

Krzesinski, J., \& Wojcik, K. 1993, Astr. Ap., 280, 338

Lacy, C. 1992, AJ, 104, 801

Maceroni, C., Biancini, A., Rodono, M., van 't Veer, F., \& Vio, R. 1990, A\&A, 237, 395

Montes, D., Fernandez-Figueroa, M., De Castro, E., \& Cordine, M. 1994, A\&A, 285, 609

Montes, D., Fernandez-Figueroa, M., De Castro, E., \& Cordine, M. 1995, A\&A, 294, 165

Morris, D., \& Mutel, R. 1988, AJ, 95, 204

Naftilan, S. 1987, AJ, 94, 1327

Oliver, J. 1974, Ph.D. Thesis, Univ. of California, Los Angeles

Perryman, M., Lidegren, L., Kovalevsky, J., et al. 1997, A\&A, 323, L49 (The Hipparcos catalogue)

Pojmanski, G. 1998, Acta Astron., 48, 711

Popper, D. 1976, IBVS, 1083

Rao, P., Sarma, M., \& Rao, B. 1991, J. Astrophys. Astron., 12, 225

Saar, S., \& Brandenburg, A. 1999, ApJ, 524, 295

Sarma, M. 1976, Bull. Astron. Inst. Czechoslovakia, 27, 335

Smirnov, O., Piskunov, N., Afanasyev, V., \& Morozov, A. 1992, in Astronomical Data Analysis Software and Systems 1, ASP Conf. Ser., 26, 344

Strassmeier, K., Hall, D., Zeilik, M., et al. 1988, A\&AS, 72, 291

Strassmeier, K., Fekel, F., Bopp, B., Dempsey, R., \& Henri, G. 1990, ApJS, 72, 191

Walter, F., Cash, W., Charles, P., \& Bowyer, C. 1980, ApJ, 236, 212

Wilson, R., \& Devinney, E. 1971, ApJ, 166, 605

Wilson, R. 1993, Dokcumentation of Eclipsing Binary Computer Model

Zarro, D., \& Rogers, A. 1983, ApJS, 53, 815

Zeilik, M., Cox, D., Ledlow, M., \& Rhodes M. 1989, IBVS, 3384

Zeilik, M., Cox, D., Ledlow, M., et al. 1990, ApJ, 363, 647 